\title{
Proximal and Distal Gastric Retention Patterns in Gastroparesis and the Impact of Gastric Per-Oral Endoscopic Myotomy: A Retrospective Analysis Using Gastric Emptying Scintigraphy
}

\author{
Robert Spandorfer*1, Yin Zhu*1, Mohamed M. Abdelfatah ${ }^{1}$, Parit Mekaroonkamol ${ }^{1}$, Sunil Dacha ${ }^{1}$, James R. Galt ${ }^{2}$, \\ Raghuveer Halkar ${ }^{2}$, and Qiang $\mathrm{Cai}^{1}$ \\ ${ }^{1}$ Division of Digestive Diseases, Emory University School of Medicine, Atlanta, Georgia; and ${ }^{2}$ Department of Radiology and Imaging \\ Sciences, Emory University School of Medicine, Atlanta, Georgia
}

\begin{abstract}
Gastroparesis is a debilitating disease of insufficient gastric emptying and visceral hypersensitivity characterized by nausea, vomiting, early satiety, and bloating. Gastric emptying scintigraphy (GES), in combination with typical symptoms and normal esophagogastroduodenoscopy findings, is used to diagnose the disease. Gastric per-oral endoscopic pyloromyotomy (G-POEM) has emerged as a novel technique for treating gastroparesis, with up to an $80 \%$ success rate. This procedure involves myotomy of the distal stomach. We hypothesize that responders to this therapy are characterized by more distal dysmotility than nonresponders, as defined by GES retention patterns. Methods: We used regional gastric emptying measurements from diagnostic GES to determine the proximal or distal predominance of disease for each patient. We then compared treatment response and symptoms in each patient to total gastric half-emptying time $\left(T_{1 / 2}\right)$, proximal gastric $T_{1 / 2}$, and a ratio comparing the 2 values. Results: In total, 47 patients underwent G-POEM during the study period. A significant difference $(P<0.01)$ was found in proximal-to-total $T_{1 / 2}$ ratio between responders and nonresponders. A significant difference between pre- and postprocedural proximalto-total $T_{1 / 2}$ ratios was identified for each patient. No correlations were identified between motility patterns and symptoms or in motility patterns among the different etiologies of the disease. Conclusion: Proximal-to-total $T_{1 / 2}$ ratio may represent an important patient selection factor for G-POEM versus other treatment modalities going forward. Local retention patterns in GES may not inform the symptom profile in gastroparesis.
\end{abstract}

Key Words: GPOEM; POP; gastroparesis; gastric emptying scintigraphy

J Nucl Med Technol 2020; 48:158-162

DOI: 10.2967/jnmt.119.235630

\footnotetext{
Received Aug. 26, 2019; revision accepted Nov. 21, 2019.

For correspondence or reprints contact: Qiang Cai, Emory University School

of Medicine, 1365 Clifton Rd., Suite B1262, Atlanta, GA 30322.

E-mail: qcai@emory.edu

${ }^{*}$ Contributed equally to this work.

Published online Dec. 6, 2019.

COPYRIGHT @ 2020 by the Society of Nuclear Medicine and Molecular Imaging.
}

$\mathbf{G}_{\mathbf{a}}$ astroparesis is a chronic disorder of gastric motility with debilitating symptoms, including nausea, vomiting, early satiety, bloating, and distension. There are multiple etiologies, including diabetic, postinfectious, postsurgical, and idiopathic. Current therapies include lifestyle and dietary modifications, medications (such as metoclopramide, domperidone, and erythromycin), and procedural therapies (such as pyloric botulinum toxin, gastric electrical stimulation, pyloroplasty, or subtotal gastrectomy), but none is particularly effective (1-3).

The condition is diagnosed using a combination of typical symptoms, a gastric emptying scintigraphy (GES) study demonstrating abnormal food retention, and normal esophagogastroduodenoscopy findings to rule out obstruction (3). GES involves consuming a radioactive tracer in the form of a meal, with imaging to examine food retention at different time points. Although this study is typically used to assess global gastric function, it may also give insight into regional gastric motility and the underlying pathophysiologic mechanisms of gastroparesis, in particular by comparing proximal and distal food retention (4-9). Specifically, half-emptying time $\left(T_{1 / 2}\right)$ has been used as a proxy for global and regional stomach function $(7,9,10)$.

Multiple therapies aimed at the distal stomach have been developed, such as pyloric botulinum toxin and pyloric stenting $(2,11,12)$. Among these distally acting therapies, gastric peroral endoscopic pyloromyotomy (G-POEM) has recently been introduced as a promising, novel therapeutic modality (13-16). This procedure involves a small endoscopic incision into the antral muscle to reduce tone and promote gastric emptying, using technical concepts similar to the per-oral endoscopic myotomy procedure for achalasia (17). Despite exciting initial results for G-POEM, a subset of patients have disease refractory to the procedure. It is currently unclear what factors predispose a patient to successful G-POEM (13-15).

The purpose of this study was to use GES to compare proximal and distal food retention patterns in patients who have undergone G-POEM and to explore correlations among 
local dysmotility patterns, symptomatology, and treatment effectiveness. We hypothesize that, as a distal therapy, it would stand to reason that G-POEM would be most likely to benefit those patients with primarily distal retention $(12,18)$. On the other hand, it is expected that those patients with a more proximal burden of disease would benefit less from this procedure. Additionally, we aimed to identify a correlation between certain retention profiles and specific symptoms.

\section{MATERIALS AND METHODS}

This was a retrospective study examining local motility patterns in patients with severe, refractory gastroparesis and the correlation with G-POEM success. Refractory disease was defined as failure of lifestyle changes and at least 2 medical therapies. The study was approved by the Institutional Review Board at Emory University. Beginning in June 2015, patients were evaluated as candidates for G-POEM, based on a protocol approved by the Institutional Review Board. All patients who received G-POEM during the study period were included; liquid, oatmeal, and anterior-imaging-only studies were excluded. Only standard and comprehensive GES studies were included.

Patients were diagnosed using a 4-h gastric scintigraphy test in most cases, and all patients were asked to undergo a follow-up GES about 2 mo after G-POEM as well. The GES used a protocol consistent with the Consensus Recommendations for Gastric Emptying Scintigraphy by the American Neurogastroenterology and Motility Society and the Society of Nuclear Medicine and Molecular Imaging (4). A ${ }^{99 \mathrm{~m} T c-s u l f u r ~ c o l l o i d ~ r a d i o l a b e l e d ~ m e a l ~ c o n s i s t i n g ~ o f ~}$ the egg-white equivalent of 2 large eggs, 2 slices of bread, and jam with water was administered. Imaging was performed in the anterior and posterior projections at $0,1,2,3$, and $4 \mathrm{~h}$. A geometric mean activity of decay-corrected counts in the 2 projections was calculated at each time point and represented the primary data used.

The G-POEM procedure was performed as previously described (15), by an advanced endoscopist who was frequently assisted by a trainee, with the patient under general anesthesia in the endoscopy suite. After a routine esophagogastroduodenoscopy, the endoscopist made a 2-cm incision into the gastric mucosa at the 5-o'clock position, approximately $5 \mathrm{~cm}$ from the pyloric ring. This incision was followed by dissection of submucosal fibers from the mucosal entry site to the pyloric ring, to form a submucosal tunnel. After myotomy inside the tunnel, the tunnel was rinsed with saline and the mucosal entry site was closed with hemostatic clips.

Outcome measures were recorded using the Gastroparesis Cardinal Symptoms Index (GCSI), with follow-up at 1, 6, 12, 18, and 24 mo when available. The GCSI is a validated tool for quantifying the severity of gastroparesis symptoms, using 3 categories and 9 subsets (19). The categories include postprandial fullness or early satiety (4 subsets), nausea or vomiting (3 subsets), and bloating (2 subsets). Each subset is scored $0-5$, with 5 for the highest severity, and each symptom category is averaged for a total GCSI score ranging from 0 to 5. This score was obtained for each patient before the procedure and was used as a primary follow-up metric. Successful treatment response was defined as a reduction in total GCSI score of 1 point, with a $25 \%$ reduction in at least 2 of the 3 subsets (15).

Data collected for each patient include demographics, etiology of disease, and the GCSI metrics described above at 1-mo, 6-mo, 12-mo, and 24-mo intervals as available.

GES studies from our institution were processed for 4-h total gastric retention as well as proximal and distal retention. Only those studies performed at our institution were used for this advanced processing; those not performed at our institution were diagnosed elsewhere and only global GES measurements were included. Regions of interest were drawn manually on a Xeleris 3 Functional Imaging Workstation (GE Healthcare). The proximal and distal stomach was defined using the incisura as an anatomic landmark for each patient, such that the proximal stomach represents the fundus and the body. Examples are shown in Figure 1. The data that were recorded include tracer retention at $0,1,2,3$, and $4 \mathrm{~h}$, as well as $\mathrm{T}_{1 / 2}$ for the total stomach, proximal stomach, and distal stomach.

The primary gastric-emptying data that were collected include total gastric, proximal gastric, and antral tracer kilocounts at each time point, as well as total gastric $T_{1 / 2}$, proximal gastric $T_{1 / 2}$, and the proximalto-total $\mathrm{T}_{1 / 2}$ ratio, which was defined as the retention index (RI) for this study; therefore, an RI of 1 denotes completely proximal retention and an RI of 0 denotes completely pyloric retention. These data were compared between responders and nonresponders and between idiopathic and diabetic disease etiologies using the Student $t$ test. A $P$ value of less than 0.05 was considered significant. These values were also compared with patient symptoms before and after the procedure and with symptom reduction from the procedure using Pearson correlations. All statistics were performed on Microsoft Excel.

\section{RESULTS}

From June 2015 through October 2017, 47 patients underwent G-POEM at our institution. Their demographics are shown in Table 1. The initial average GCSI was 3.52, with an SD of 0.69. Most patients responded to G-POEM therapy.

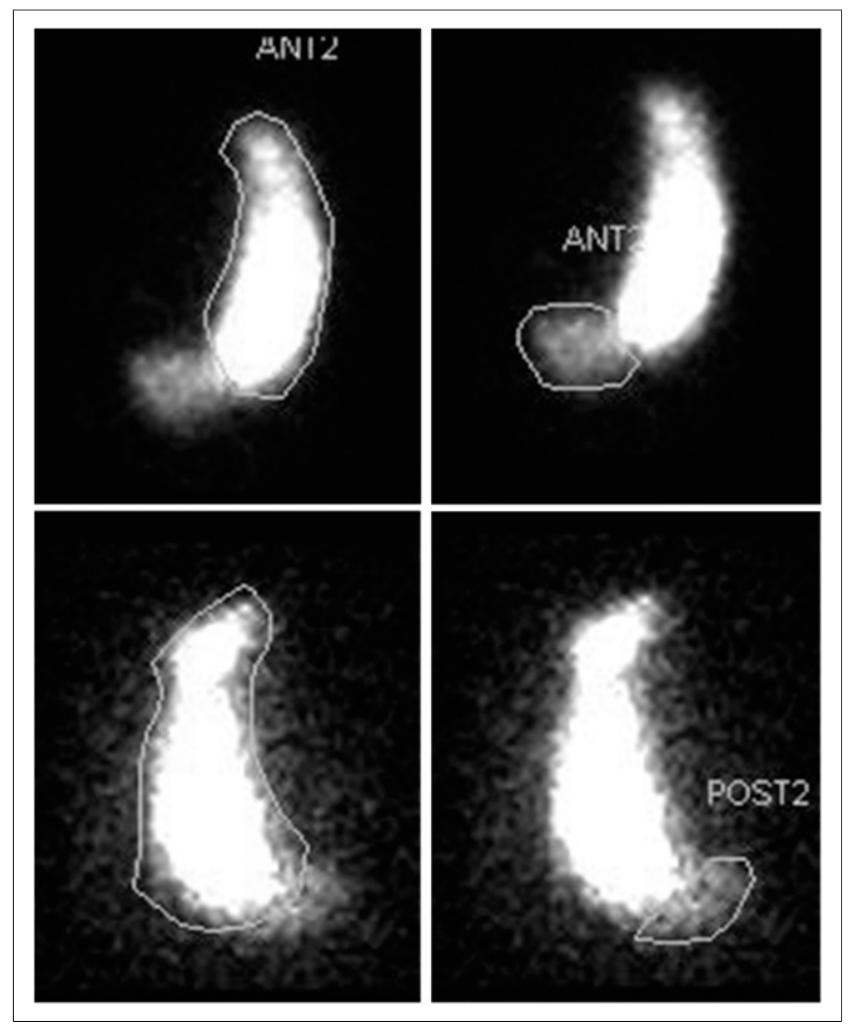

FIGURE 1. Example of ROI selection, using incisura as anatomic landmark. (Top left) Proximal stomach in anterior view. (Top right) Distal stomach in anterior view. (Bottom left) Proximal stomach in posterior view. (Bottom left) Distal stomach in posterior view. 
TABLE 1

Patient Demographics by Etiology

\begin{tabular}{lcccc}
\hline \multicolumn{1}{c}{ Etiology } & Male & Female & Total & Age $(\mathrm{y})$ \\
\hline Idiopathic & 1 & 18 & 19 & $43 \pm 12$ \\
\hline Diabetic & 1 & 14 & 15 & $48 \pm 16$ \\
Postsurgical & 2 & 3 & 5 & $66 \pm 11$ \\
\hline Postinfectious & 0 & 2 & 2 & $29 \pm 7$ \\
After radiotherapy & 1 & 0 & 1 & 52 \\
Ehlers-Danlos & 0 & 1 & 1 & 33 \\
\hline Unrecorded & 1 & 3 & 4 & $54 \pm 6$ \\
Total & 6 & 41 & 47 & $47 \pm 15$
\end{tabular}

$\mathrm{XRT}=$ radiation therapy.

Age is expressed as average \pm SD.

Among the 47 patients, there were 12 preprocedure GES and 19 postprocedure GES studies; 5 patients had GES studies from both before and after the procedure. The Grubbs test for a single outlier was run for the preprocedural total and proximal $T_{1 / 2}$ data, and 1 additional patient was excluded with a $P$ value of less than 0.001 . For the postprocedural total and proximal gastric $T_{1 / 2}$ data, the Iglewicz and Hoaglin robust test for multiple outliers was run for a modified $z$ score of at least 3.5, and 2 additional patients were identified as outliers and excluded. The outlier patients' data were incompatible with the remainder of the cohort's data or what would be expected, suggesting an improperly performed test or measurement error. An exclusion flowchart is shown in Figure 2.

For all included patients, RI (the proximal-to-distal gastric $T_{1 / 2}$ ratio) was calculated both before and after the procedure.

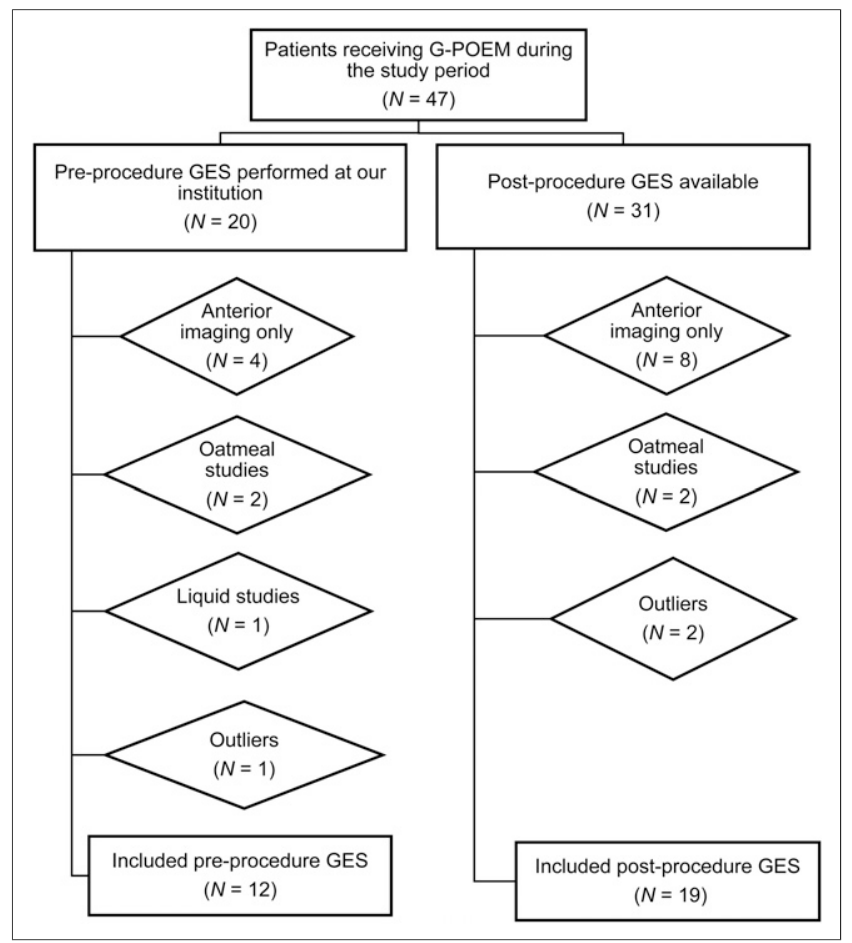

FIGURE 2. Flowchart reflecting patient exclusion from this study.
TABLE 2

Average Preprocedural RI by Etiology

\begin{tabular}{lc}
\hline Etiology & Average preprocedural RI \\
\hline Idiopathic & $0.877 \pm 0.12$ \\
Diabetic & $0.872 \pm 0.052$ \\
Total & $0.880 \pm 0.090$
\end{tabular}

$\mathrm{RI}$ is expressed as average $\pm \mathrm{SD}$.

The preprocedural RI by etiology is shown in Table 2. No significant difference in RI was found between diabetic and idiopathic disease.

RI values did not inform the symptom profile, as presented in Figure 3.

Five patients (all of whom were responders to G-POEM) had GES studies performed at our institution and were available for localized measurement both before and after the procedure. A 0.06 decrease in RI was found, which was shown to be statistically significant using a 2-tailed, paired Student $t$ test. These data are represented in Table 3.

Eleven patients ( 10 responders, 1 nonresponder) had total $T_{1 / 2}$ available before and after the procedure. Within this group, the preprocedural total gastric $\mathrm{T}_{1 / 2}$ averaged 206.6, with an SD of 75.2; the postprocedural total gastric $\mathrm{T}_{1 / 2}$ averaged 122.9, with a SD of 36.7. This represents a reduction in $T_{1 / 2}$ of $83.7 \mathrm{~min}$ after the procedure. This reduction in gastric $\mathrm{T}_{1 / 2}$ was statistically significant, with a $P$ value of 0.0028 .

There were 23 responders and 16 nonresponders at the latest available follow-up date. All other patients were not available for follow-up. Response rates did not significantly differ by etiology.

The preprocedural RI for responders ranged from 0.847 to 1.056 , with an average of 0.924 and an SD of 0.0686 . The preprocedural RI for nonresponders ranged from 0.705

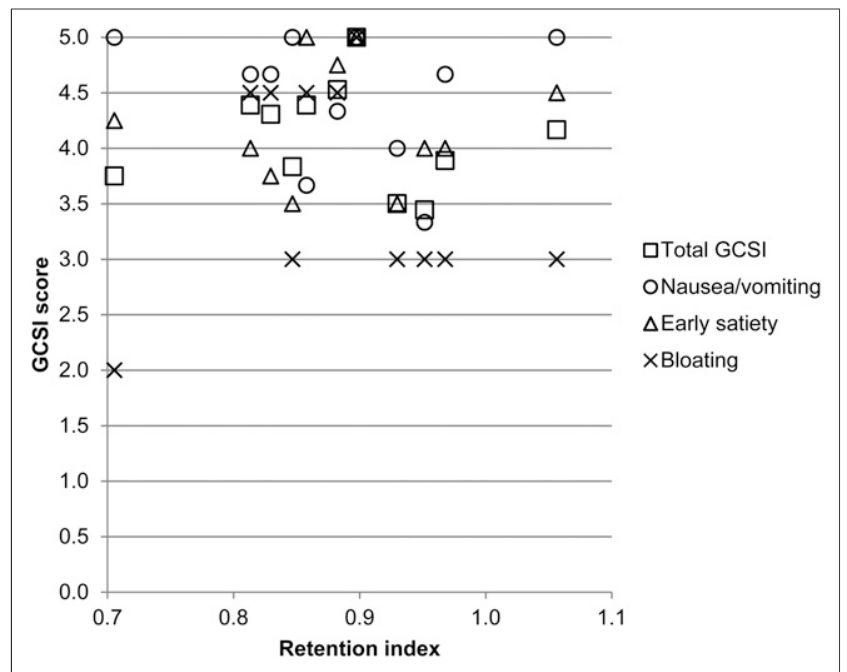

FIGURE 3. Total GCSI and symptom categories vs. RI for each patient. 
TABLE 3

RI Before and After G-POEM in Patients with Both Studies Available

\begin{tabular}{lcc}
\hline Patient no. & RI before G-POEM & RI after G-POEM \\
\hline 1 & 0.973 & 0.847 \\
2 & 1.052 & 0.952 \\
3 & 1.090 & 1.057 \\
4 & 0.882 & 0.858 \\
5 & 0.936 & 0.898 \\
Average \pm SD & $0.987 \pm 0.0847$ & $0.922 \pm 0.0856$ \\
\hline
\end{tabular}

to 0.829 , with an average of 0.794 and an SD of 0.0593 . This represents an RI difference of 0.13 between the 2 groups. The 2 averages were compared using a 2-tailed $t$ test, yielding a $P$ value of less than 0.01 .

A comparison of RI and GCSI reduction for each patient is displayed in Figure 4.

The postprocedural RI and reduction in RI for each patient were compared with total GCSI and each symptom category using Pearson correlations. These Pearson correlations were all nonsignificant, with values of less than 0.01 .

When total gastric $T_{1 / 2}$ and proximal $T_{1 / 2}$ were compared with GCSI reductions, all Pearson correlations were less than 0.01 . No significant difference was found in total gastric $T_{1 / 2}$, proximal $T_{1 / 2}$, or postprocedural RI between responders and nonresponders. The results are shown in Table 4.

\section{DISCUSSION}

The results of this study indicate that there is no major difference in RI among the many etiologies of gastroparesis. Diabetic and idiopathic gastroparesis are the 2 most common etiologies, both in this study and in the general population, and these 2 etiology groups had a nearly identical preprocedural RI, with no significant difference between the two. The response rates between these 2 groups were similar as well, suggesting that these etiologies of gastroparesis may follow similar pathophysiologic mechanisms because they have similar retention patterns and response rates. The fact that local motility patterns and response rates to the procedure are

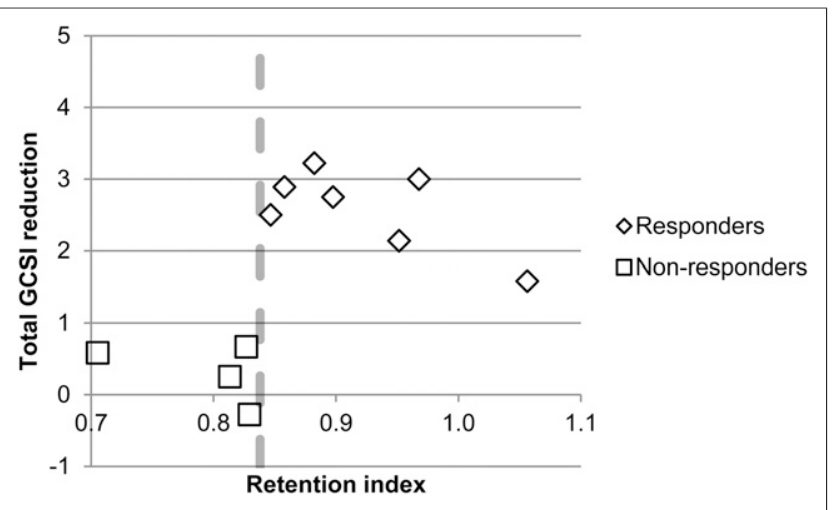

FIGURE 4. GCSI reduction vs. preprocedural RI. Dashed line represents $R I$ value of 0.838 .
TABLE 4

Absolute $T_{1 / 2}$ Compared Between Responders and Nonresponders

\begin{tabular}{lccl}
\hline \multicolumn{1}{c}{ Parameter } & Responders & Nonresponders & $P$ \\
\hline Before G-POEM TG T & $169 \pm 42$ & $218 \pm 80$ & 0.11 \\
Before G-POEM PG T $_{1 / 2}$ & $149 \pm 37$ & $141 \pm 27$ & 0.68 \\
After G-POEM TG T $_{1 / 2}$ & $131 \pm 42$ & $108 \pm 37$ & 0.31 \\
Before G-POEM RI & $0.924 \pm 0.069$ & $0.794 \pm 0.059$ & 0.006 \\
After G-POEM RI & $0.924 \pm 0.070$ & $0.886 \pm 0.070$ & 0.19
\end{tabular}

$\mathrm{TG}=$ total gastric emptying. $\mathrm{PG}=$ proximal gastric emptying

Data are average \pm SD. There were insufficient complete data to compare $P G T_{1 / 2}$ after G-POEM between responders and nonresponders.

nearly identical between these 2 etiologies indicates that etiology should not be a major factor when considering who may benefit most from this procedure. All postsurgical patients responded to the procedure. There were not enough postsurgical patients with available preprocedural GES to calculate a meaningful average RI.

The next finding is that there was no obvious correlation between local motility and symptomatology, as expressed by the near-zero Pearson correlations between symptom score and RI, both before and after the procedure. This finding indicates that RI is not a useful metric for predicting a patient's symptoms. Indeed, some studies have suggested that proximal and distal dysfunction independently correlate with different symptom profiles for gastroparesis or functional dyspepsia $(5,20-23)$. However, neither proximal nor distal motility measurements in GES corresponded to symptoms in this study.

Our analysis shows a significant change in RI for each patient after the procedure. In fact, every patient with available data had an increase in RI. This finding demonstrates that the procedure is achieving a clear and significant effect on gastric emptying; an increase in RI indicates either an increase in proximal $T_{1 / 2}$ or a decrease in total gastric $T_{1 / 2 .}$. Considering that the procedure acts distally, the latter mechanism is much more likely. This possibility was confirmed, as we also demonstrated a clear reduction in total gastric $\mathrm{T}_{1 / 2}$ after the procedure, suggesting that the procedure functions in a manner similar to our hypothesis: a reduction of tone lessens retention, decreasing total gastric $T_{1 / 2}$, increasing RI, and promoting emptying.

We showed a clear and significant difference in preprocedural RI between responders and nonresponders. Although statistically significant, this was a very small dataset of 5 patients; however, as a novel procedure with limited research thus far, these few patients still represent a valuable cohort size. In our study, the responder groups demonstrated a significantly higher RI or a more proximal retention pattern. This difference is the opposite of what we expected: an antral therapy should provide the greatest benefit to those patients with primarily distal disease.

It is unclear why those patients with more proximal disease would receive the greatest benefit from therapy. One possibility is that rather than a longer $T_{1 / 2}$ in the proximal stomach, 
these patients are actually demonstrating a faster emptying in the entire stomach, perhaps indicating less severe disease at presentation. It would stand to reason that patients with less severe motility defects are less likely to be refractory to the procedure. However, no significant difference in absolute proximal or total $\mathrm{T}_{1 / 2}$ between the 2 groups was identified.

Another possibility is that although the procedure decreases pyloric tone, it may simultaneously disrupt antral motility. One primary mechanism of gastroparesis that has been suggested is pylorospasm, which is often coupled with antral hypomotility (12,24). Assuming this mechanism, patients with a more distal burden of disease may have greater antral hypomotility at baseline. They would thus be more sensitive to further antral disruption. Patients with a more proximal burden of disease may be less likely to have significant antral dysmotility before the procedure and would thus be less sensitive to this negative sequela. Further research into this concept is necessary for us to understand the pathophysiology of gastroparesis and how the G-POEM procedure works on a functional basis.

Regardless of the underlying pathophysiology, RI demonstrates significant promise as a patient-selection factor going forward. In fact, all patients in the responder group had an RI greater than 0.838 , whereas all patients in the nonresponder group had a value lower than this number, or a more distal pattern of retention. This finding is displayed in Figure 4, with the dashed line representing this cutoff. A prospective study comparing outcomes of patients with RI greater than and less than that value would be important to identify appropriate candidates for this procedure and help further develop the current gastroparesis treatment algorithm.

\section{CONCLUSION}

Our study found that patients with a more proximal burden of gastroparesis are more likely to benefit from G-POEM. The calculated RI based on local gastric motility measurements shows promise as a tool to determine who should receive this procedure but warrants further investigation before clinical implementation. Some limitations of this study include the small sample size and a lack of healthy control patients. Because this is a novel procedure, there is a limited number of patients available for study. This problem must be addressed as the procedure gains popularity and more gastroenterologists are trained to perform it. Additionally, for many patients, only GES studies from outside institutions were available to us, and we could not process those studies for local motility measurements. Although the sample size was small, our results are significant and suggest that further research into this topic across multiple centers may yield promising results for future patient selection. This investigation involved only patients with gastroparesis, who by definition will have GES abnormalities. A more thorough analysis is needed to compare these patients' motility values with those of healthy controls. Finally, the retrospective and single-center nature of this trial brings limitations, and a more robust, prospective analysis should be performed.

\section{DISCLOSURE}

No potential conflict of interest relevant to this article was reported.

\section{REFERENCES}

1. Camilleri M. Clinical practice: diabetic gastroparesis. N Engl J Med. 2007;356: 820-829.

2. Hejazi RA, McCallum RW. Treatment of refractory gastroparesis: gastric and jejunal tubes, Botox, gastric electrical stimulation, and surgery. Gastrointest Endosc Clin N Am. 2009;19:73-82.

3. Stein B, Everhart KK, Lacy BE. Gastroparesis: a review of current diagnosis and treatment options. J Clin Gastroenterol. 2015;49:550-558.

4. Abell TL, Camilleri M, Donohoe K, et al. Consensus recommendations for gastric emptying scintigraphy: a joint report of the American Neurogastroenterology and Motility Society and the Society of Nuclear Medicine. Am J Gastroenterol. 2008;103:753-763.

5. Gonlachanvit S, Maurer AH, Fisher RS, Parkman HP. Regional gastric emptying abnormalities in functional dyspepsia and gastro-oesophageal reflux disease. Neurogastroenterol Motil. 2006;18:894-904

6. Bennink R, Peeters M, Van den Maegdenbergh V, et al. Comparison of total and compartmental gastric emptying and antral motility between healthy men and women. Eur J Nucl Med. 1998;25:1293-1299.

7. Jones KL, Horowitz M, Wishart MJ, Maddox AF, Harding PE, Chatterton BE. Relationships between gastric emptying, intragastric meal distribution and blood glucose concentrations in diabetes mellitus. J Nucl Med. 1995;36:2220-2228.

8. Orthey P, Yu D, Van Natta ML, et al. Intragastric meal distribution during gastric emptying scintigraphy for assessment of fundic accommodation: correlation with symptoms of gastroparesis. J Nucl Med. 2018;59:691-697.

9. Guo WJ, Yao SK, Zhang YL, Yan J, Yin LJ, Li HL. Relationship between symptoms and gastric emptying of solids in functional dyspepsia. J Int Med Res. 2012;40:1725-1734.

10. Kotani K, Kawabe J, Kawamura E, et al. Clinical assessment of delayed gastric emptying and diabetic complications using gastric emptying scintigraphy: involvement of vascular disorder. Clin Physiol Funct Imaging. 2014;34:151-158.

11. Jones MP, Maganti K. A systematic review of surgical therapy for gastroparesis. Am J Gastroenterol. 2003;98:2122-2129.

12. Gourcerol G, Tissier F, Melchior C, et al. Impaired fasting pyloric compliance in gastroparesis and the therapeutic response to pyloric dilatation. Aliment Pharmacol Ther: 2015;41:360-367.

13. Gonzalez JM, Benezech A, Vitton V, Barthet M. G-POEM with antro-pyloromyotomy for the treatment of refractory gastroparesis: mid-term follow-up and factors predicting outcome. Aliment Pharmacol Ther. 2017;46:364-370.

14. Khashab MA, Ngamruengphong S, Carr-Locke D, et al. Gastric per-oral endoscopic myotomy for refractory gastroparesis: results from the first multicenter study on endoscopic pyloromyotomy (with video). Gastrointest Endosc. 2017;85:123-128.

15. Dacha S, Mekaroonkamol P, Li L, et al. Outcomes and quality-of-life assessment after gastric per-oral endoscopic pyloromyotomy (with video). Gastrointest Endosc. 2017;86:282-289.

16. Rodriguez JH, Haskins IN, Strong AT, et al. Per oral endoscopic pyloromyotomy for refractory gastroparesis: initial results from a single institution. Surg Endosc. 2017;31:5381-5388.

17. Smith SP, Louie BE. The current state of per oral endoscopic myotomy for achalasia. J Vis Surg. 2017;3:122.

18. Wellington J, Scott B, Kundu S, Stuart P, Koch KL. Effect of endoscopic pyloric therapies for patients with nausea and vomiting and functional obstructive gastroparesis. Auton Neurosci. 2017;202:56-61.

19. Revicki DA, Rentz AM, Dubois D, et al. Gastroparesis Cardinal Symptom Index (GCSI): development and validation of a patient reported assessment of severity of gastroparesis symptoms. Qual Life Res. 2004;13:833-844.

20. Piessevaux H, Tack J, Walrand S, Pauwels S, Geubel A. Intragastric distribution of a standardized meal in health and functional dyspepsia: correlation with specific symptoms. Neurogastroenterol Motil. 2003;15:447-455.

21. Maurer AH. Advancing gastric emptying studies: standardization and new parameters to assess gastric motility and function. Semin Nucl Med. 2012;42:101-112.

22. Karamanolis G, Caenepeel P, Arts J, Tack J. Determinants of symptom pattern in idiopathic severely delayed gastric emptying: gastric emptying rate or proximal stomach dysfunction? Gut. 2007;56:29-36.

23. Camilleri M. Novel diet, drugs, and gastric interventions for gastroparesis. Clin Gastroenterol Hepatol. 2016;14:1072-1080.

24. Geyl S, Legros R, Charissou A, et al. Peroral endoscopic pyloromyotomy accelerates gastric emptying in healthy pigs: proof of concept. Endosc Int Open. 2016;4: E796-E799. 